\title{
Unstable mixing regions in II-VI quaternary solid solutions
}

\author{
V.G. Deibuk, S.G. Dremlyuzhenko, S.E. Ostapov \\ Yu. Fed'kovich Chernivtsi National University, 2, Kotsyubinsky str., 58012 Chernivtsi, Ukraine \\ Phone: +38-03722-44500; e-mail:vdei@chnu.cv.ua
}

\begin{abstract}
The miscibility gaps and critical temperatures of the spinodal decompositions of the quaternary semiconducting epitaxial thin films CdMnHgTe and $\mathrm{ZnMnHgTe}$ have been calculated. Fitting the spinodal isotherms calculated from the free energy of mixing being based on application of the delta-lattice parameter method to the experimental data, the model constant $K$ is determined. It has been shown the narrowing of the spinodal decomposition regions due to both the deformation energy and the effect of plastic relaxation caused by mismatch dislocations in thin films.
\end{abstract}

Keywords: quaternary alloys, spinodal decomposition, II-VI thin films.

Manuscript received 19.08.05; accepted for publication 25.10.05.

\section{Introduction}

Semiconductor devices fabricated on the basis of II-VI compounds and the corresponding solid solutions find recently a wide practical application in solid-state electronics. The interest in multicomponent alloys that are potentially very important materials for the production of infra-red detectors, low-noise filters of communication systems, and continuously tunable lasers, has appreciably increased. CdHgTe alloys are the most accessible from semiconductor materials currently used in manufacturing the devices for the IR spectral range [1]. However, up to the data most advanced semiconductor devices are based on the ability to grow single crystalline film materials with the variation of important properties such as bandgap in the growth direction and should have no changes in lattice constant, i.e., the bandgap and the lattice constant must be independently specified. This generally requires a semiconductor alloy with two compositional degrees of freedom, namely, quaternary alloys. The existence of miscibility gaps in quaternary II-VI alloys leads to the tendency for clustering or spinodal decomposition.

The technological difficulties of growing CdMnHgTe and ZnMnHgTe solid solutions [2] hampered its wide introduction into practice until recently. However, the recent achievements in this area [3-5] opened new prospects for its production and application. Moreover, it is worth noting the growing interest in such fundamental characteristics as electronic band structure [6], transport [4], magnetic [7], optical [5] properties of specified materials.

In spite of the fact that the issues of thermodynamic stability of the examined solid solutions are of the great practical interest, the relevant detailed calculations have not been carried out till now.

In this paper, we report the results of studying the influence of the mismatch dislocation formation in thin epitaxial films on the regions of thermodynamic stability making use of the delta-lattice parameter (DLP) model [8]. The effects of biaxial deformations induced by the generation of mismatch dislocations in the specified thin epitaxial films have been analyzed.

\section{Elastic energy in spinodal decomposition processes}

Except for the analysis of the dependences of the gap width and the lattice constants on the composition of a solid solution, it is necessary to take into account the fact that most of quaternary solid solutions are unstable in the relevant range of composition. A solid solution in the region of instability tends to diminish its free energy through decomposition, i.e., a phase transformation, which results in a violation of the crystal macroscopical homogeneity and the appearance of a mixture of phases with different compositions. Disordered semiconductor alloys have a positive enthalpy of mixing, which leads to a decomposition and counteracts the stabilizing action of internal strains. Moreover, disorder of multinary II-VI solid solutions manifests two tendencies upon phase transformations, namely, the decay and formation of superstructures [9].

The regions of immiscibility and instability that exist in many ternary and almost all quaternary $\mathrm{A}^{\mathrm{II}} \mathrm{B}^{\mathrm{VI}}$ solid solutions substantially limit the range of compositions that can be used for making devices. As was shown in [10], strains and deformations can lead to an extension of the solubility region. Deformation provides also an 
additional degree of freedom for the adjustment of the band structure and a monitored modification of optical and kinetic properties of semiconductors. Deformations and strains in materials grown by epitaxial methods, such as molecular-beam epitaxy (MBE), metal organic chemical vapor deposition (MOCVD), etc., are caused mainly by the lattice mismatch between an epitaxial layer and a substrate. Elastic relaxation in thin films also affects phase transformations and stability. It is necessary to note that, upon the spinodal decomposition, the purely coherent matching of two phases opposes the conjugation processes and can be accompanied by the appearance of mismatch dislocations [11]. A realization of each specified opportunity depends on the spinodal decomposition kinetics.

For the thermodynamic description of the pseudoternary $\mathrm{A}_{\mathrm{x}} \mathrm{B}_{\mathrm{y}} \mathrm{C}_{1-\mathrm{x}-\mathrm{y}} \mathrm{D}$ solid solutions, consider the Gibbs free energy of mixing per mole:

$\Delta G=\Delta H-T \Delta S$,

where $\Delta H$ is enthalpy of mixing, $T$ is the absolute temperature, $\Delta S$ is the entropy of mixing that may be written down as

$\Delta S=-R k\{x \ln x+y \ln y+$

$+(1-x-y) \ln (1-x-y)\}$.

For the description of the enthalpy of mixing, we use DLP model, in which [12]

$\Delta H=E($ alloy $)-x E(A D)-y E(B D)-$

$-(1-x-y) E(C D)=K\left\{a_{\text {alloy }}^{-2.5}-x a_{A D}^{-2.5}-\right.$

$\left.-y a_{B D}^{-2.5}-(1-x-y) a_{C D}^{-2.5}\right\}$,

where $K$ is the model parameter. The spinodal is defined by the condition [13]:

$\left(\frac{\partial^{2} G}{\partial x^{2}}\right)\left(\frac{\partial^{2} G}{\partial y^{2}}\right)-\left(\frac{\partial^{2} G}{\partial x \partial y}\right)^{2}=0$.

In the case where solid solution is a thin epitaxial film, and the thermodynamic process runs by the mechanism of formation of mismatch dislocation, biaxial deformations will arise in the film due to a mismatch of the lattice constants of the solid solution, $a_{\text {alloy, }}$, and the substrate material, $a_{\text {sub }}$. Then, $\varepsilon_{x x}=\varepsilon_{y y}$ for the deformations in the film plane, and the normal $z$ component of the strain tensor may be written for cubic crystal as

$\tau_{z}=C_{12} \varepsilon_{x x}+C_{12} \varepsilon_{y y}+C_{11} \varepsilon_{z z}$,

where $C_{11}$ and $C_{12}$ are the elastic constants of the film. Taking into account that $\tau_{z}=0$ for a free (growthrelated) direction of the film, relation (5) yields

$\varepsilon_{z z}=-\frac{2 C_{12}}{C_{11}} \varepsilon_{x x}(x, y)$.
The energy of such deformation per unit volume can be written,

$E_{s}=\frac{E \varepsilon^{2}}{1-v}$

where $\varepsilon=\varepsilon_{x x}, E$ is Young's modulus, and $v$ is Poisson's ratio. Eq. (7) was obtained in [14] as a special case. In addition, as was shown in [15] for the first time, the elastic deformation energy for an epitaxial films changed due to the relaxation of elastic strain on the free surface. In this case, it will be more correctly to write the relation (7) as:

$E_{S}^{\prime}=\left(\frac{C_{11}}{2\left(C_{11}+C_{12}\right)}\right) \frac{E \varepsilon^{2}}{1-v}$.

The relative deformation of an entirely strained epitaxial film is

$\varepsilon(x, y)=\varepsilon_{m}(x, y)=\frac{a_{\text {alloy }}(x, y)-a_{\text {sub }}}{a_{\text {sub }}}$.

The dependence of the lattice constant $a_{\text {alloy }}(x, y)$ on the composition was described by Vegard's rule.

Nevertheless, the described situation is observed only in the case where the thickness of film $h$ is smaller than the critical one $h_{c}$. But if $h>h_{c}$, the plastic relaxation and the formation of the mismatch dislocations occur in the film. The thicker is the film, the smaller is its residual strain. To define the influence of the specified effects on the thermodynamic stability of the considered solid solutions, we note that, according to the force balance model [16], the residual strain is inversely proportional to the thickness of the epilayer:

$\varepsilon=A / h$.

The parameter $A$ is determined from the continuity condition of the function $\varepsilon(h)$ at the point $h=h_{c}$. So $A=\varepsilon_{m} h_{c}$. Most of semiconductor heteroepitaxial objects are grown on the surface (001) of a substrate, and here we shall examine just this orientation. In our calculations, we used the analytical expression of the epilayer critical thickness [16]:

$h_{c}=\left(\frac{b}{\varepsilon_{m}}\right) \frac{1}{8 \pi(1+v)}\left[\ln \left(\alpha \frac{h_{c}}{b}\right)+\beta\right]$.

where $\alpha=4, \beta=1[16], b$ is the Burgers vector modulus. Since the $60^{\circ}$-mismatch dislocations in the plane $(001)$ are the most common in semiconductor epilayers, the Burgers vectors are $(a / 2)\langle 110\rangle$, so that $b=a / \sqrt{2}$. In the dislocation core area, the strains are too large to be described correctly in the framework of the linear theory of elasticity; therefore, the phenomenological parameter $\beta$ serves as the measure of such a deviation [17]. 
Thus, the total Gibbs free energy of the system per unit volume is a sum of the chemical energy $\Delta G$ and strain energy $E_{s}{ }^{\prime}$ :

$$
G=N_{v} \Delta G+E_{s}^{\prime}
$$

where $N_{v}$ is the number of moles per volume of homogeneous solid solution before the decomposition.

The analysis of the Gibbs free energy as a function of the solid solution composition and the thickness of the epilayer, together with the stability criterion, allows one to calculate the range of the miscibility gap.

\section{Results and discussion}

The unstable mixing regions for $\mathrm{Cd}_{1-\mathrm{x}-\mathrm{y}} \mathrm{Mn}_{\mathrm{x}} \mathrm{Hg}_{\mathrm{y}} \mathrm{Te}$ and $\mathrm{Zn}_{1-\mathrm{x}-\mathrm{y}} \mathrm{Mn}_{\mathrm{x}} \mathrm{Hg}_{\mathrm{y}} \mathrm{Te}$ were simulated as follows. The boundaries between unstable and metastable regions of mixing can be expressed using spinodal isotherms. These isotherms may be calculated as inflection points for a Gibbs free-energy surface plotted as a function of $X$ and $Y$. The proportionality constant of $K$ in (3) is determined by fitting the spinodal isotherms to experimental data of respective bulk ternary alloys [18]. Therefore, $3 \times 10^{8} \mathrm{cal} / \mathrm{mol} \AA^{2.5}$ was adopted as a $K$ value. The spinodal isotherms calculated using $K$ value obtained above for $\mathrm{Cd}_{1-\mathrm{x}-\mathrm{y}} \mathrm{Mn}_{\mathrm{x}} \mathrm{Hg}_{\mathrm{y}} \mathrm{Te}$ and $\mathrm{Zn}_{1-\mathrm{x}-\mathrm{y}} \mathrm{Mn}_{\mathrm{x}} \mathrm{Hg}_{\mathrm{y}} \mathrm{Te}$ at $t=650{ }^{\circ} \mathrm{C}$ are shown in Figs 1 and 2 , respectively.

The region surrounded by the $\mathrm{CdTe}(\mathrm{ZnTe})-\mathrm{MnTe}$ line, MnTe-HgTe line and isotherms shows the unstable compositions of $\mathrm{Cd}_{1-\mathrm{x}-\mathrm{y}} \mathrm{Mn}_{\mathrm{x}} \mathrm{Hg}_{\mathrm{y}} \mathrm{Te}$ (Fig. 1) and $\mathrm{Zn}_{1-\mathrm{x}-\mathrm{y}} \mathrm{Mn}_{\mathrm{x}} \mathrm{Hg}_{\mathrm{y}} \mathrm{Te}$ (Fig. 2). These figures show the spinodal isotherms for bulk alloy (solid line), and pseudomorphic films $(h=0.1 \mu \mathrm{m})$ on CdTe (dashed line) and $\mathrm{Cd}_{0.96} \mathrm{Zn}_{0.04} \mathrm{Te}$ (dotted line) substrates. Fig. 1 also presents experimental points from different papers $[2,3,5,6,18]$ obtained from epitaxial layers deposition by LPE and MOVPE techniques, demonstrating good

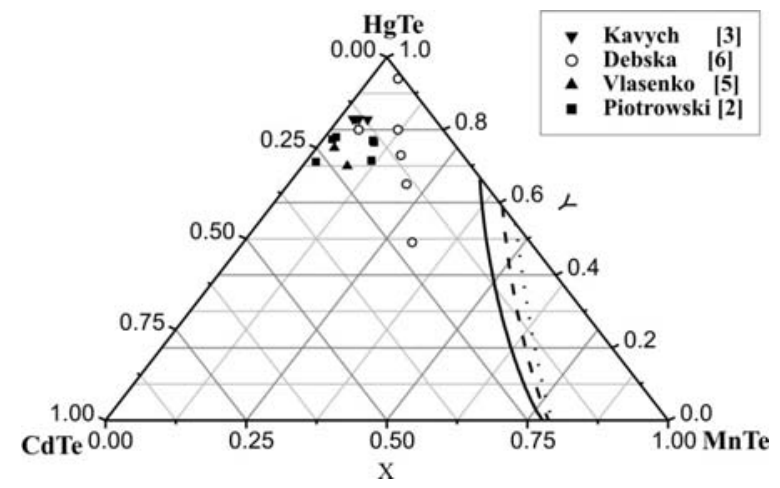

Fig. 1. Calculated spinodal isotherms for the $\mathrm{Cd}_{1-\mathrm{x}-\mathrm{y}} \mathrm{Mn}_{\mathrm{x}} \mathrm{Hg}_{\mathrm{y}} \mathrm{Te}$ system at $650{ }^{\circ} \mathrm{C}$ for a bulk alloy (solid line) and pseudomorphic films $(h=0.1 \mu \mathrm{m}) \mathrm{Cd}_{1-\mathrm{x}-\mathrm{y}} \mathrm{Mn}_{\mathrm{x}} \mathrm{Hg}_{\mathrm{y}} \mathrm{Te} / \mathrm{CdTe}$ (dashed line), $\mathrm{Cd}_{1-\mathrm{x}-\mathrm{y}} \mathrm{Mn}_{\mathrm{x}} \mathrm{Hg}_{\mathrm{y}} \mathrm{Te} / \mathrm{Cd}_{0.96} \mathrm{Zn}_{0.04} \mathrm{Te}$ (dotted line).

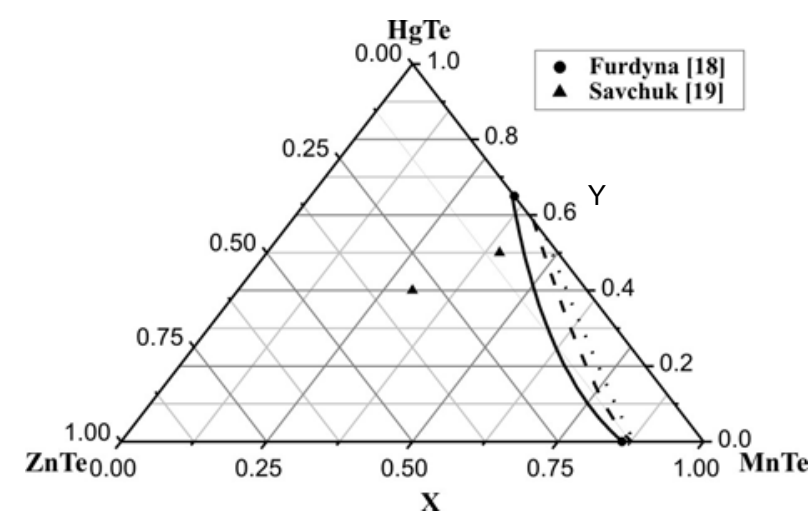

Fig. 2. Calculated spinodal isotherms for the $\mathrm{Zn}_{1-\mathrm{x}-\mathrm{y}} \mathrm{Mn}_{\mathrm{x}} \mathrm{Hg}_{\mathrm{y}} \mathrm{Te}$ system at $650{ }^{\circ} \mathrm{C}$ for a bulk alloy (solid line) and pseudomorphic films $(h=0.1 \mu \mathrm{m}) \quad \mathrm{Zn}_{1-\mathrm{x}-\mathrm{y}} \mathrm{Mn}_{\mathrm{x}} \mathrm{Hg}_{\mathrm{y}} \mathrm{Te} / \mathrm{CdTe}$ (dashed line), $\mathrm{Zn}_{1-\mathrm{x}-\mathrm{y}} \mathrm{Mn}_{\mathrm{x}} \mathrm{Hg}_{\mathrm{y}} \mathrm{Te} / \mathrm{Cd}_{0.96} \mathrm{Zn}_{0.04} \mathrm{Te}$ (dotted line).

agreement with the calculations, i.e., no experimental points fall in the thermodynamic instability region at the epitaxy temperature. When the layer thickness decreases, the strain effect increases and the miscibility gap narrows. The changes of the instability region also due to different mismatch strain of substrates (CdTe or $\left.\mathrm{Cd}_{0.96} \mathrm{Zn}_{0.04} \mathrm{Te}\right)$. Note that the situation on strained $\mathrm{Zn}_{1-\mathrm{x}-\mathrm{y}} \mathrm{Mn}_{\mathrm{x}} \mathrm{Hg}_{\mathrm{y}} \mathrm{Te}$ epilayers (Fig. 2) varies weakly.

\section{References}

1. A. Rogalski, Infrared detectors at the beginning of the next millennium // Sensors and Materials 12, p. 233-288 (2000).

2. T. Piotrowski, Preparation of $\mathrm{Hg}_{1-\mathrm{x}-\mathrm{y}} \mathrm{Cd}_{\mathrm{x}} \mathrm{Mn}_{\mathrm{y}} \mathrm{Te}$ crystals by isothermal growth method // J. Crystal Growth 72, p. 117- 119 (1985).

3. V.J. Kavych, M.I. Lozynska, L.G. Mansurov, Epitaxial MnCdHgTe layers by RF sputtering in mercury plasma // Appl. Phys. 3, p. 65-68 (2003) (in Russian).

4. O.O. Bodnaruk, S.E. Ostapov, I.M. Rarenko, M.D. Tymochko, Investigation of transport phenomena in HgMnTe and HgCdMnTe monocrystals // $J$. Alloys and Compounds 371, p. 93-96 (2004).

5. O.I. Vlasenko, V.M. Babentsov, Z.K. Vlasenko, A.V. Ponedilok, I.V. Kurilo, I.O. Rudyj, V.V. Kremenitskij, Distribution of components in epitaxial graded band gap heterostructures $\mathrm{Cd}(\mathrm{Mn}, \mathrm{Zn}) \mathrm{Te}$ $\mathrm{Cd}(\mathrm{Mn}, \mathrm{Zn}) \mathrm{HgTe}$ and their photoelectrical properties // Semiconductor Physics, Quantum Electronics and Optoelectronics 1, p. 75-81 (1998).

6. U. Debska, M. Dietl, G. Grabecki, E. Janik, E. Kierzek-Pecold and M. Klimkiewicz, Energy band gaps of $\mathrm{Hg}_{\mathrm{x}} \mathrm{Cd}_{\mathrm{y}} \mathrm{Mn}_{\mathrm{z}} \mathrm{Te}(\mathrm{x}+\mathrm{y}+\mathrm{z}=1)$ epitaxial layers // Phys. status solidi (a) 64, p. 707-713 (1981). 
7. J.C. Woolley, A. Manoogian, R.J.W. Hodgston and G. Lamarche, ESR linewidth and RKKY exchange in small band gap $\mathrm{Cd}_{\mathrm{x}} \mathrm{Hg}_{\mathrm{y}} \mathrm{Mn}_{\mathrm{z}} \mathrm{Te}$ alloys // J. Magn. Magn. Mat. 78, p. 164-170 (1989).

8. G.B. Stringfellow, Calculation of regular solution interaction parameters in semiconductor solid solutions // J. Phys. Chem. Solids 34, p. 1749-1751 (1973).

9. A. Rogalski, Infrared detectors: status and trends // Progr. in Quant. Electronics 27, p. 59-210 (2003).

10. V.G. Deibuk, S.G. Dremliuzhenko, S.E. Ostapov, A thermodynamic stability of bulk and epitaxial $\mathrm{CdHgTe}, \mathrm{ZnHgTe}$, and MnHgTe solid solutions // Semiconductors 39, p. 1120-1125(2005).

11. J.H. Van der Merve, W.A. Jesser, An exactly solvable model for calculating critical misfit and thickness in epitaxial superlattices // J. Appl. Phys. 63, p. 1509-1517(1988).

12. A. Chen, A. Sher, Semiconductor alloys: physics and material engineering. Plenum Press, New York (1995).
13. G.B. Stringfellow, Spinodal decomposition and clustering in III/V alloys // J. Electron. Mater. 11, p. 903-918 (1982).

14. L.D. Landau, E.M. Lifshits, Theory of elasticity. Nauka, Moskow (1985) (in Russian).

15. I.P. Ipatova, V.G. Malyshkin, V.A. Shchukin, On spinodal decomposition in elastically anisotropic epitaxial films of III-V semiconductor alloys // J. Appl. Phys. 74, p.7198-7210(1993).

16. R. Beanland, D.J. Dunstan, P.J. Goodhew, Plastic relaxation and relaxed buffer layers for semiconductor epitaxy // Adv. Phys. 45, p. 87-146 (1996).

17. V.G. Deibuk, The thermodynamic stability of GaInSb, InAsSb, and GaInP epitaxial films // Semiconductors 37, p. 1151-1155 (2003).

18. J.K. Furdyna, Diluted magnetic semiconductors // J. Appl. Phys. 64, p. R29-R63 (1988).

19. A.I. Savchuk, V.I. Fediv, V.M. Frasuniak, I.D. Stolyarchuk, P.I. Nikitin, Enhancement of magneto-optical effects in ZnHgMnTe // J. Crystal Growth 197, p. 698-701 (1999). 